\title{
Effect of alloy type and casting technique on the fracture strength of implant-cemented structures
}

\author{
Raquel Castillo-de Oyagüe ${ }^{\text {, }}$, Raquel Osorio ${ }^{2}$, Christopher Lynch ${ }^{3}$, Alan Gilmour ${ }^{4}$, Manuel Toledano ${ }^{5}$
}

${ }^{1}$ DDS, PhD, Associate Professor. Department of Prosthodontics. Faculty of Dentistry. Complutense University of Madrid, UCM, 28040 Madrid, Spain

${ }^{2}$ DDS, PhD, Professor. Department of Dentistry, School of Dentistry. University of Granada (UGR), Campus de la Cartuja, 18071, Granada, Spain

${ }^{3}$ BDS, PhD, MFD, RCSI, FDS (Rest Dent), RCSI, Senior Lecturer/Consultant, Department of Adult Dental Health, School of Dentistry, Cardiff University, CF14 4XY, Cardiff, Wales, UK

${ }^{4}$ BDS, FDSRCS (Ed), PhD, Reader/Consultant, Department of Adult Dental Health, School of Dentistry, Cardiff University, CF14 4XY, Cardiff, Wales, UK

${ }^{5}$ MD, DDS, PhD, Professor. Department of Dentistry, School of Dentistry. University of Granada (UGR), Campus de la Cbleartuja, 18071, Granada, Spain

Correspondence:

Department of Prosthodontics.

Faculty of Dentistry.

Complutense University of Madrid, U.C.M.

Pza. Ramón y Cajal s/n, 28040 Madrid, Spain

raquel.castillo@odon.ucm.es

Received: 05/06/2010

Accepted: 25/07/2010

Castillo-de Oyagüe R, Osorio R, Lynch C, Gilmour A, Toledano M.. Effect of alloy type and casting technique on the fracture strength of implant-cemented structures. Med Oral Patol Oral Cir Bucal. 2011 Jul 1;16 (4):e619-25.

http://www.medicinaoral.com/medoralfree01/v16i4/medoralv16i4p619.pdf

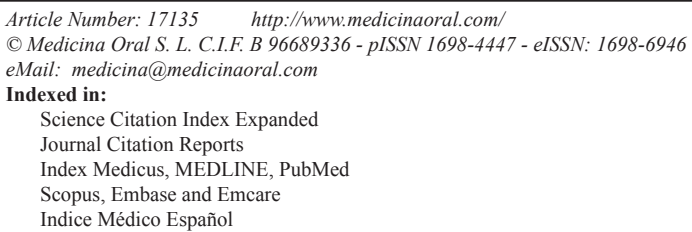

\begin{abstract}
Objectives: To evaluate the influence of alloy type and casting procedure on the fracture strength (FS) of metallic frameworks for implant-supported fixed prostheses.

Study design: Thirty three-unit structures for lower posterior bridges were waxed-up and randomly assigned to two groups $(\mathrm{n}=15)$ according to alloy type and casting technique: Group 1 (C): cobalt-chromium cast in a centrifugal machine (TS1, Degussa-Hüls); Group 2 (T): titanium cast in a pressure-differential device (Cyclarc II, Morita). Each structure was cemented onto two prefabricated abutments under a constant seating pressure. After 6 months of water aging, samples were loaded in a static universal testing machine (EFH/5/FR, Microtest) until fracture. Axial compressive loads were applied at the central fossa of the pontics. FS data were recorded and surface topography of the fractured connectors was SEM-analyzed. A Chi-Square test was performed to assess the dependence of pores on the alloy type and casting procedure. ANOVA and Student-Newman-Keuls (SNK) tests were run for FS comparisons $(\mathrm{p}<0.05)$.

Results: One third of the $\mathrm{C}$ structures showed pores inside the fractured connectors. $\mathrm{T}$ frameworks demonstrated higher FS than that of $\mathrm{C}$ specimens exhibiting pores $(\mathrm{p}=0.025)$. $\mathrm{C}$ samples containing no pores recorded the greatest mean FS $(\mathrm{p}<0.001)$.

Conclusions: Fracture strength of metallic frameworks depended on the alloy type and casting procedure. Cobaltchromium casts often registered pores inside the connectors, which strongly decreased the fracture resistance. An accurate casting of titanium with a pressure-differential system may result in the most predictable technique under the tested experimental conditions.
\end{abstract}

Key words: Metallic framework, titanium, cobalt-chromium, casting technique, fracture strength. 


\section{Introduction}

The biomechanical behavior of implant-retained restorations may be compromised by, amongst others, accumulated errors in the casting procedure (1). Cast frameworks may suffer distortion, marginal misfit, and/or airentrapment, thus enhancing the effects of mechanical fatigue $(2,3)$. Interactions of such factors might lead to catastrophic failures, either under repeated loading, or after stress concentrations exceeding the ultimate strength of the materials. Although little scientific data have been published on the performance of cobaltchromium alloys for implant-prosthetic applications (4), cobalt-chromium structures are the most widely used in implant dentistry due to their high fracture strength, elastic module and hardness (5), absence of potential allergenic and carcinogenetic components (such as nickel and beryllium) and low cost (6). In contrast, titanium has become more popular in implant-supported superstructures due to its superior strength-to-weight ratio, recognized biocompatibility and corrosion resistance $(1$, $6,7)$. Notwithstanding the difficulty of casting titanium alloys, usually involving special investment materials, high melting points and an inert atmosphere (1), little research has been conducted concerning the precision of recently-marketed casting units for titanium in comparison with traditional centrifugal devices $(7,8)$.

Despite the accuracy of CAD/CAM systems for fabricating metallic structures, casting technologies continue to be more frequently used largely because of economic reasons (1). However, very few investigations have focused on the inherent physical properties of differently processed frameworks $(9,10)$. Given the complexity in achieving a perfect cast with current technologies (2), this study aimed to indirectly evaluate the castability of cobalt-chromium and pure titanium by determining the fracture strength and interfacial morphology of the fractured surfaces of cast superstructures. Moreover, no previous research has been carried out on this topic.

The null hypothesis tested was that there are no differences in the fracture resistance and topographic patterns of the fracture fragments of implant-bridge cemented frameworks regardless the alloy type (cobalt-chromium vs. pure titanium) and their associated investing and casting techniques (centrifugal cast vs. pressure-differential system, respectively).

\section{Materials and Methods}

\section{-Experimental design and casting techniques}

Two conical titanium abutments for cemented restorations (height: $6 \mathrm{~mm}$ ) (ref. PCM7013, Implant Microdent System, Barcelona, Spain) were connected to their corresponding implant replicas (diameter: $3.8 \mathrm{~mm}$ ) and torqued to $35 \mathrm{Ncm}$. Abutments were fixed into a special aluminum platform using stainless steel screws and type IV plaster. Two series of structures were waxed and cast with different alloys. Chemical composition of the tested metals is described in Table 1. The frameworks consisted of three-unit posterior-lower bridgestructures for implant-cemented prostheses with an intermediate pontic (spanning the first premolar to the first molar). Wax patterns (Bego-Dental Wax, Bremen, Germany) were prepared over burnout casting copings (ref. CCM7011 - Implant Microdent System, Barcelona, Spain), and randomly assigned to two groups ( $\mathrm{n}=15$ each).

Group 1 (C) was cast using a base metal alloy of white cobalt-chromium for ceramics (IPS d.SIGN30, Ivoclar Vivadent, Madrid, Spain). Patterns were invested in

Table 1. Chemical composition of the alloys tested in the study.

\begin{tabular}{|c|c|}
\hline Dental alloys & Composition [weight (wt) \%)] \\
\hline $\begin{array}{l}\text { Cobalt-chromium } \\
\text { (IPS d.SIGN30, IvoclarVivadent) }\end{array}$ & $\begin{array}{l}\text { cobalt ( } 60.2 \text { wt } \%) ; \\
\text { chromium }(30.1 \mathrm{wt} \%) ; \\
\text { gallium (3.9 wt \%); } \\
\text { niobium (3.2 wt \%); } \\
\text { traces of: silicium, molybde- } \\
\text { num, boron, iron, aluminum and } \\
\text { lithium. }\end{array}$ \\
\hline $\begin{array}{l}\text { Pure type II titanium } \\
\text { (Titan 15, Morita) }\end{array}$ & $\begin{array}{l}\text { titanium }(99,794 \text { wt } \%) ; \\
\text { traces of: oxygen, ferrum, car- } \\
\text { bonum, nitrogen and hydrogen. }\end{array}$ \\
\hline
\end{tabular}


cylinders without a metallic ring, using a phosphatebased improved plaster (Deguvest-Impact, DegussaHüls, Hanau, Germany). The cast was performed in a centrifugal machine (TS1, Degussa-Hüls, Frankfurt, Germany) at $1220^{\circ} \mathrm{C}$.

Group 2 (T) was cast with type II pure titanium base metal for ceramics (Titan 15, J. Morita, Kyoto, Japan). An alumina-magnesia-system was used as investment material (Titavest CB, J. Morita, Kyoto, Japan). The "A $+\mathrm{C}$ " mode was programmed in the pressure-differential machine (Cyclarc II, J. Morita, Kyoto, Japan). The casting device consisted on two-chamber pressure/vacuum system that smelts the titanium at the temperature of $1700{ }^{\circ} \mathrm{C}$ with a voltaic arc under an inert argon atmosphere.All bridge structures were carefully removed and sandblasted with $50 \mu \mathrm{m}$ aluminum-oxide particles for $10 \mathrm{~s}$ at a working distance of $5 \mathrm{~mm}$ and a pressure of 60-100 psi.

\section{-Luting procedure}

Cementation was performed to simulate clinical conditions. Metallic frameworks were luted to their respective abutments with Temp Bond (Kerr, Oklahoma, USA). A special clamp was designed to maintain a constant seating pressure of $25 \mathrm{Ncm}$ for $4 \mathrm{~min}$. Two hexagon-shaped relieves (fitting perfectly into the abutment's hexagons and duplicating their position in the initial metallic support) were incorporated on the clamp basis. Axial surfaces of the abutments placed in the clamp were varnished with a thin cement layer before inserting each bridge structure. The clamp press was unscrewed until it contacted with the specimen's occlusal surface. The upper screw that controlled the press was fitted with a torque driver tool for implant applications (Defcon I-72000, Impladent, Barcelona, Spain). Bonded specimens were stored in distilled water at $37^{\circ} \mathrm{C}$ and $100 \%$ humidity for six months before Fracture Strength (FS) testing.

\section{-Fracture strength test}

Based on a previous FS protocol that aimed to simulate in vivo conditions (11), thirty custom-made blocks of composite material (Tetric Evo Ceram, A3, batch no J27435, Ivoclar-Vivadent; Schaan, Liechtenstein) were fabricated using a cubic-shaped silicon mould. The implant-replicas of every bridge framework were embedded inside a composite cube. This procedure was carried out to reproduce the mean elastic module (E) reported for trabecular human bone $\left[\mathrm{E}_{\text {(Tetric) }}=15,1+/-0.8\right.$ $\mathrm{GPa}(12) ; \mathrm{E}_{\text {(Bone) }}=13.5$ +/- $2.0 \mathrm{GPa}$ (13)]. Composite increments were condensed around the implant-replicas with a clean plastic instrument to avoid contamination, and light-cured for $40 \mathrm{~s}$ (BluePhase, Ivoclar-Vivadent, output: $600 \mathrm{mmW} / \mathrm{cm}^{2}$ ). Once removed from the mould, an extra $40 \mathrm{~s}$ irradiation was performed on the portions of the cubes that had previously been in contact with the silicone patterns. After 24h, all samples were test- ed for fracture strength (FS) in a computer-supported universal testing machine $(\mathrm{EFH} / 5 / \mathrm{FR}$, Microtest S.A.; Madrid, Spain) at a cross-head speed of $1 \mathrm{~mm} / \mathrm{min}$. The composite cubes containing the bridge structures were placed and fixed to the basis of the testing device. Axial compressive static loads were applied at the occlusal fossa of the pontics. Loading was applied until spontaneous rupture, and failure forces were recorded with the computer software (SCM4000, Microtest S.A.; Madrid, Spain). The fracture initiation point was determined by a sudden drop in the loading curve and perceived as a loud cracking sound. Fracture strength values (FS) were expressed in Newton $(\mathrm{N})$. Both retainers of every bridge structure were numbered using an indelible marking pen to identify the alloy group and specimen. Once retrieved from the composite cubes and implant replicas, the fractured pieces still bonded to the abutments were gently ultrasonicated for $1 \mathrm{~h}$ and air dried.

-Fracture pattern analysis

Failure modes were evaluated by a single operator under an optical microscope (BH-2 Olympus; Tokyo, Japan) at $60 \times$ magnifications, and classified as ductile (plastic deformation and tearing of metal) or brittle (no significant plastic deformation showing flat surfaces at the fracture line). Percentages of fractured structures containing pores were recorded per tested group.

-Statistical procedures

Normal fracture strength data distribution was confirmed by Kolmogorov-Smirnov test, and homogeneity of variances was verified according to Levene's test. A Chi-Square test was performed to assess the dependence of pores on the alloy type and casting procedure. One-way analysis of variance (ANOVA) and StudentNewman-Keuls (SNK) tests were run for FS comparisons considering the pore presence as a discriminating factor. The significance level was set at $\alpha=0.05$.

-Scanning Electron Microscopy (SEM) evaluation

Surface topography of representative fractured connectors was assessed using scanning electron microscopy (SEM, JSM-6400, Jeol, Tokyo, Japan). Specific areas were explored, focusing with different magnifications (from $20 \times$ to $1500 \times$ ) to better analyze the fractured connectors' surfaces.

\section{Results}

Mean and standard deviation fracture strength (FS) values recorded in the tested groups are summarized in Table 2. The alloy type and its associated investing and casting technique significantly affected FS of metallic frameworks $(p<0.0001)$. Interaction between factors -alloy type and casting method vs. pore presence-was significant.

One third of the $\mathrm{C}$ structures showed pores inside the fractured connectors. $\mathrm{T}$ frameworks demonstrated higher FS than that of $\mathrm{C}$ specimens exhibiting pores 


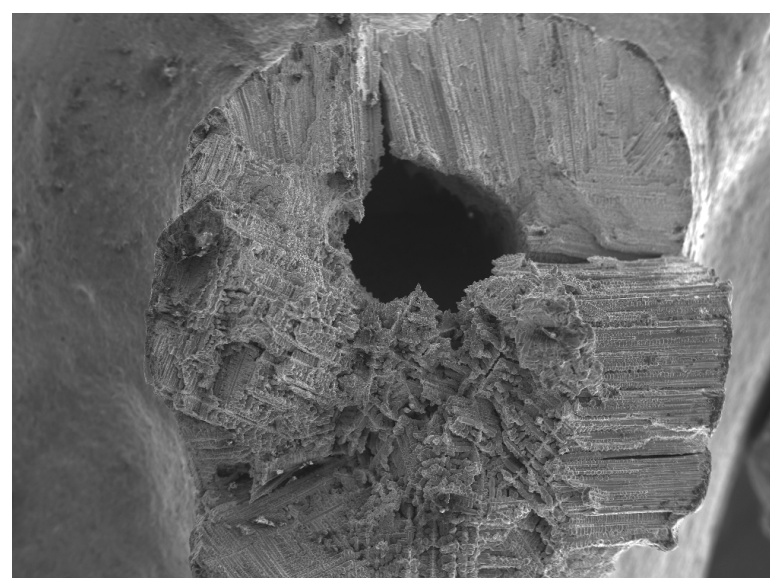

Fig. 1. A) SEM micrographs of a cobalt-chromium fractured connector containing a large pore. A stepped and rough topography coexists with a smoother surface around the hole. (a) $25 \times$ magnifications; $20 \mathrm{kV}$; bar $2 \mathrm{~mm}$.

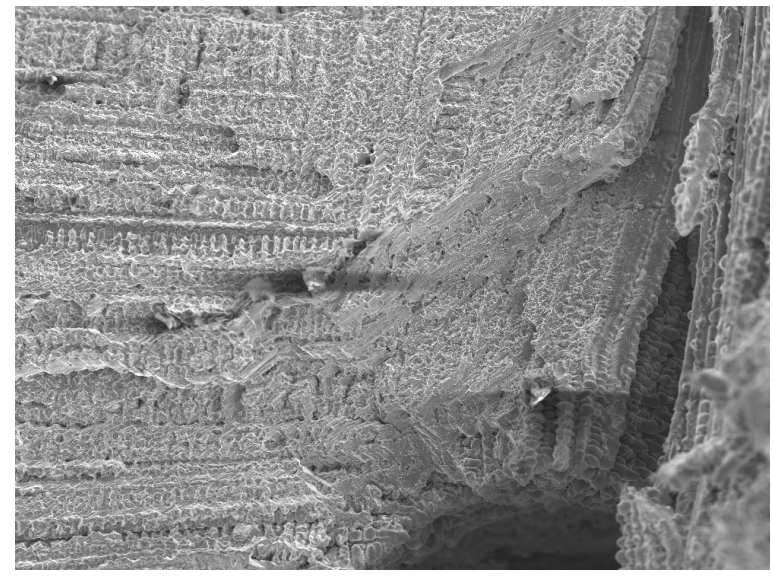

Fig. 1. B) $100 \times$ magnifications; $20 \mathrm{kV}$; bar $500 \mu \mathrm{m}$.

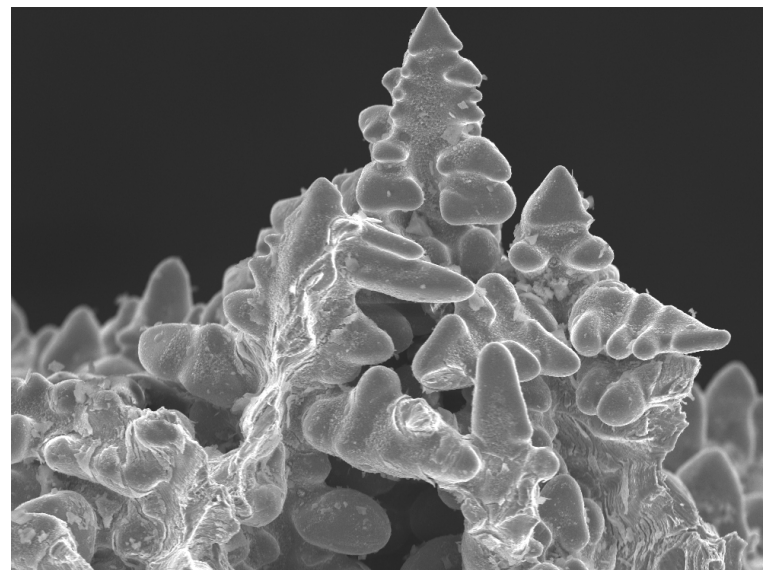

Fig. 1. C) $500 \times$ magnifications; $20 \mathrm{kV}$; bar $100 \mu \mathrm{m}$.
Table 2. Percentages of pores inside the bridge connectors, and mean (SD) values of fracture resistance $(\mathrm{N})$ recorded in the experimental groups, are displayed in the table.

\begin{tabular}{|c|c|c|c|}
\hline \multicolumn{4}{|c|}{ Pore presence (\%) and Fracture Resistance (N) } \\
\hline $\begin{array}{l}\text { Alloy type and } \\
\text { casting process }\end{array}$ & \multicolumn{2}{|c|}{$\begin{array}{c}\text { Pore presence } \\
\text { (\% of structures) }\end{array}$} & $\begin{array}{c}\text { Fracture resistance } \\
\text { Mean (SD) }\end{array}$ \\
\hline & Yes & $33.4 \%$ & $3407.2(408.53) \mathrm{c}$ \\
\cline { 2 - 4 } $\begin{array}{l}\text { Cobalt-chromium } \\
\text { (Centrifugal casting) }\end{array}$ & No & $66.6 \%$ & $8361.7(672.99) \mathrm{b}$ \\
\hline $\begin{array}{l}\text { Titanium } \\
\text { (Pressure-differential } \\
\text { casting) }\end{array}$ & Yes & $0 \%$ & \\
\hline & No & $100 \%$ & 4308.14 (776.56) a \\
\hline
\end{tabular}

$(\mathrm{p}=0.025)$. However, $\mathrm{C}$ samples containing no pores recorded the greatest mean FS $(\mathrm{p}<0.001)$.

A ductile failure was identified in $100 \%$ of the specimens, so that the higher plastic deformation detected at the ultimate fracture strength, the rougher surfaces at the broken connectors. As a consequence of the jagged topography, the fragments could not be perfectly repositioned at the fractured line, mainly in case of $\mathrm{C}$ structures containing pores (Fig. 1.A).

Representative SEM images of the experimental groups are displayed in Figures 1-3. The $\mathrm{C}$ fractured connector containing the grossest pore detected in the study (diameter: $1 \mathrm{~mm}$ ) is shown in Figure 1, surrounded by long notches. Two different microstructures could be identified around the hole. The rough stepped topography corresponded to deformed regions (Figs. 1.A, 1.C). Conversely, a bit scratched but flat surface was located at the least distorted sector (Fig. 1a). However, regularly oriented striations were discovered in this area at higher magnification (Fig. 1.B).

A fractured connector of a $\mathrm{C}$ specimen containing no porosities can be observed in Figure 2. Rough surfaces with differently-oriented striations were detectable (Figs. 2.A, 2.B). Sectors showing irregularities and deeper fractured lines were evident at higher magnification (Fig. 2.C).

$\mathrm{T}$ fractured structures exhibited the highest density and more organized fine striations (Fig. 3). At progressive higher magnifications, the striations seemed to be dissipated, showing a dense plane surface (Figs. 3.B, 3.C). 


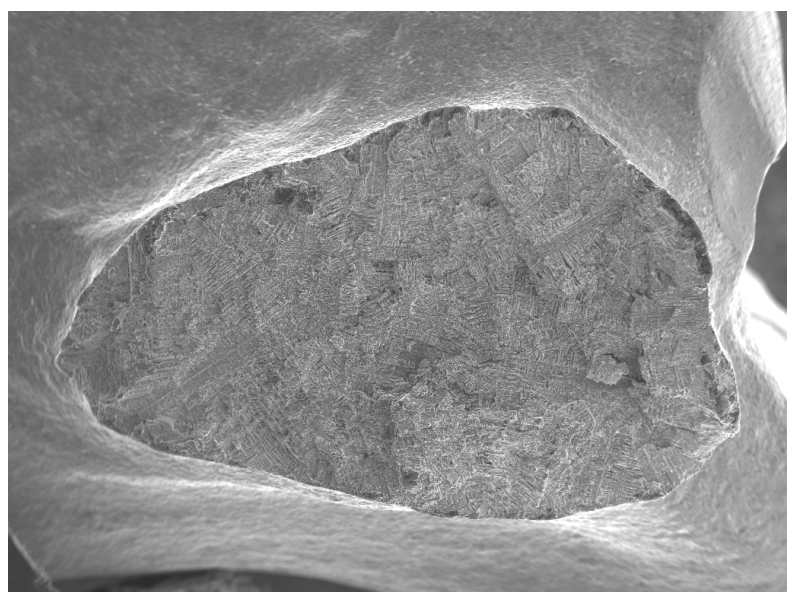

Fig. 2. A) SEM images of a cobalt-chromium fractured connector showing no porosities. An unparallel striated pattern with a fibrous appearance at different fracture levels is evident. (a) $20 \times$ magnifications; $20 \mathrm{kV}$; bar $2 \mathrm{~mm}$.

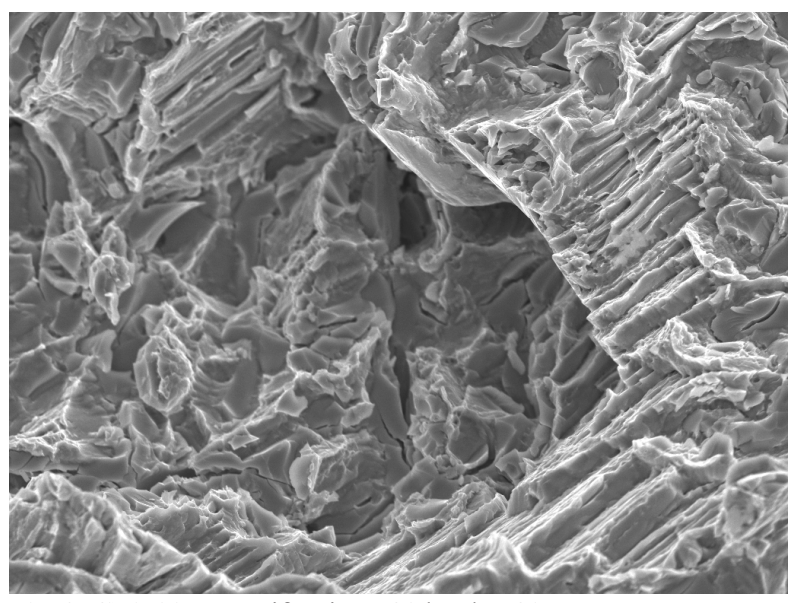

Fig. 2. C) $1500 \times$ magnifications; $20 \mathrm{kV}$; bar $30 \mu \mathrm{m}$.

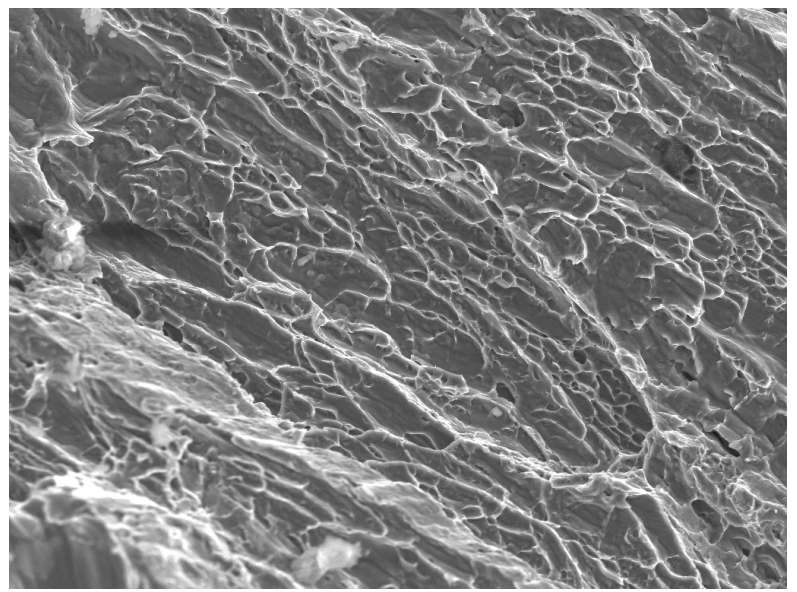

Fig. 3. B) $500 \times$ magnifications; $20 \mathrm{kV}$; bar $100 \mu \mathrm{m}$.

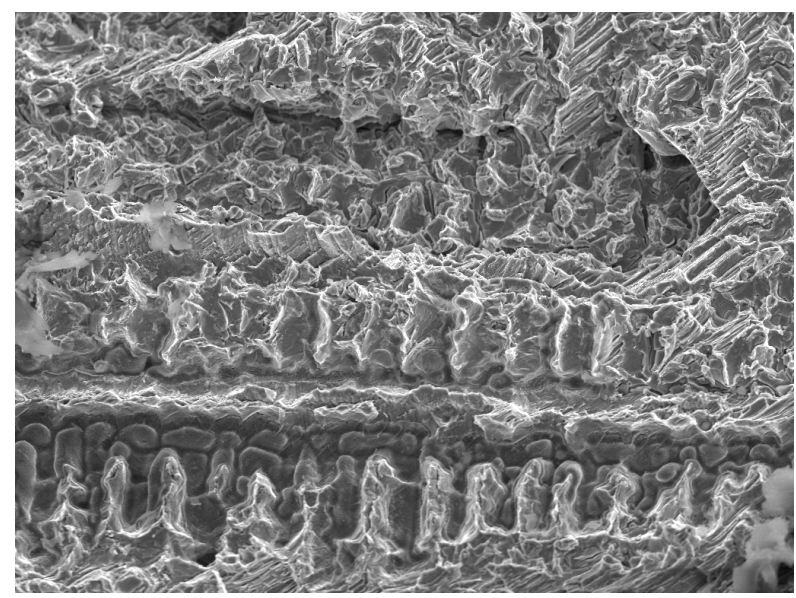

Fig. 2. B) $500 \times$ magnifications; $20 \mathrm{kV}$; bar $100 \mu \mathrm{m}$.

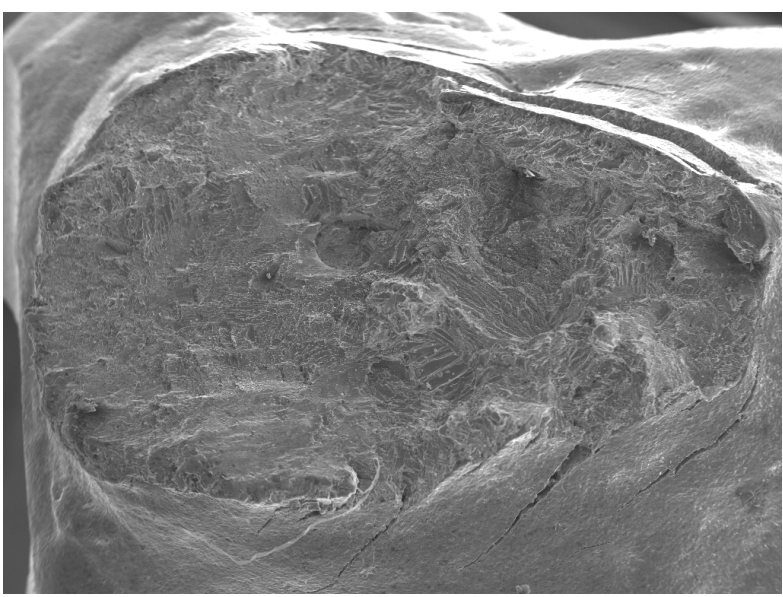

Fig. 3. A) SEM micrographs of a titanium fractured connector. A higher density and flatter surface is noticeable. (a) $20 \times$ magnifications; $20 \mathrm{kV}$; bar $2 \mathrm{~mm}$.

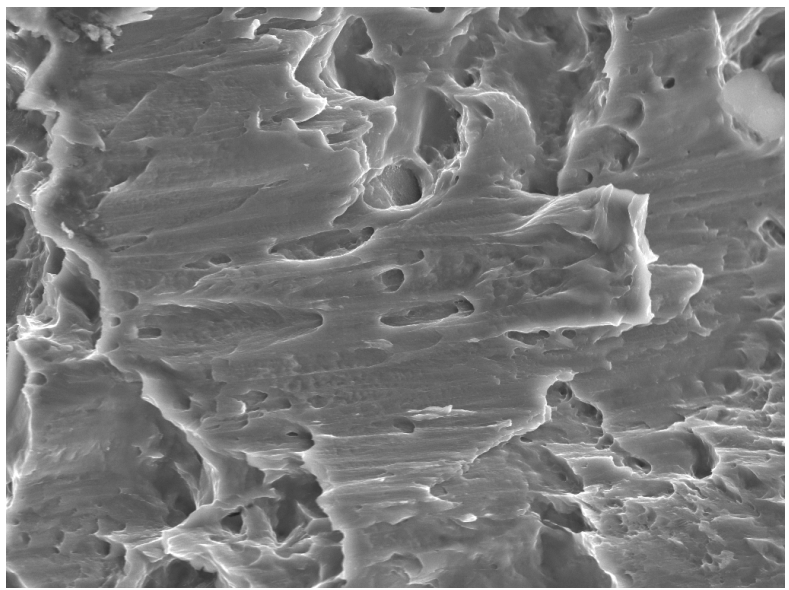

Fig. 3. C) $1500 \times$ magnifications; $20 \mathrm{kV}$; bar $30 \mu \mathrm{m}$. 


\section{Discussion}

A number of alloys may be chosen for fabricating implant-supported cemented superstructures. However, concerns still remain regarding the identification of the best alloy composition and casting technology (2). The purpose of the present research was to compare two dental alloys according to their required casting methods, by assessing the ultimate load and interfacial morphology of the fractured fragments of bridge frames luted onto two implant abutments. The findings of this study require the rejection of the null hypothesis; since differences in fracture strength and topographic patterns were recorded (Table 2) (Figs. 1-3). The cobalt-chromium alloy cast in a centrifugal machine resulted in the least predictable option in terms of fracture strength due to the remarkable frequency of air entrapment inside the connectors. Despite the highest mean fracture resistance being recorded for cobalt-chromium structures containing no pores, porosity defects detected in one third of the cobalt-chromium frames (Fig. 1) hampered their fracture strength when compared to that of the titanium structures (Table 2). Nonetheless, all the specimens tested reached greater ultimate loads than the mean occlusal forces reported for implant-supported prostheses in the oral cavity (144N) (9). Even the maximum expectable forces -that occur in the molar region in cases of parafunctional activity such as bruxism-, range from $500 \mathrm{~N}$ to $880 \mathrm{~N}(14)$.

Wictorin et al. (15) analyzed the frequency and site of internal defects in sixty-six cobalt-chromium cast frameworks by using a non-destructive X-ray method. As a result, 294 pores or cracks were recorded in sixtyfour structures. Surprisingly, very little research on this topic has been made since then. Minor percentages of pores were detected in the present study even when a SEM was used. Improvements in investing and casting technologies in the last decades may be explaining reasons.

Jang et al. (7) obtained comparable porosity for both cobalt-chromium and titanium in cast structures for removable partial dentures. Obvious differences concerning the frameworks' design and the casting devices used for cobalt-chromium (Optivest, Degussa) and for titanium (Rematitan, Dentaurum) make data comparison difficult. Bessing and Bergman (16) tested the castability of pure titanium in different machines, so that the most accurate structures were those cast in the Cyclarc pressure-differential device. These findings may support the predictability of results obtained in the present experiment for the titanium group.

Nowadays it can be argued from the infrequency of catastrophic metal failures as a result of chewing or clenching forces, that the problem is no longer serious (17). However, lesser metal fatigue issues, often unrecognized, such as plastic distortions in presence of porosity defects can promote cracking of the veneering ceramics (18). Depending on the location, amount, distribution and diameter size of the inner porosities a fracture of the metallic substructure itself may occur. When the defects are situated in a critical region - i.e. connectors-, large pores or significant porosity may result in a fracture (15) either after repeated functional loading or from intense stress locations. In this regard, the lack of screws in implant-cemented superstructures has been reported to turn even small discrepancies into static loads, resulting in mechanical deformations and stress concentrations (18). Therefore, the clinical success of metal-ceramic restorations also depends on the integrity of the cast structures. In absence of pores, minimum tensile stresses may be transferred to the veneering ceramics because of the high elastic moduli of both cobalt-chromium and titanium metal substrates $(17,19)$.

As a result, not only are the physical properties of metals used important, but also the predictability of quality of the obtained frameworks. This study suggests that selecting the most appropriate and predictable dental alloy and casting technique is a crucial factor in the long term success of the restoration. Titanium is inherently difficult to cast because of its high melting temperature, strong affinity with oxygen, hydrogen and nitrogen, as well as its high reactivity with most investment materials (1). However, a high-quality precision casting of titanium has been reported to improve the accuracy of the

Table 2. Percentages of pores inside the bridge connectors, and mean (SD) values of fracture resistance $(\mathrm{N})$ recorded in the experimental groups, are displayed in the table.

\begin{tabular}{|l|c|c|c|}
\hline \multicolumn{4}{|c|}{ Pore presence (\%) and Fracture Resistance (N) } \\
\hline \multicolumn{1}{|c|}{$\begin{array}{c}\text { Alloy type and } \\
\text { casting process }\end{array}$} & \multicolumn{2}{|c|}{$\begin{array}{c}\text { Pore presence } \\
\text { (\% of structures) }\end{array}$} & $\begin{array}{c}\text { Fracture resistance } \\
\text { Mean (SD) }\end{array}$ \\
\hline $\begin{array}{l}\text { Cobalt-chromium } \\
\text { (Centrifugal casting) }\end{array}$ & Yes & $33.4 \%$ & $3407.2(408.53) \mathrm{c}$ \\
\cline { 2 - 5 } & No & $66.6 \%$ & $8361.7(672.99) \mathrm{b}$ \\
\hline $\begin{array}{l}\text { Titanium } \\
\text { (Pressure-differential casting) }\end{array}$ & Yes & $0 \%$ & - \\
\hline & No & $100 \%$ & $4308.14(776.56) \mathrm{a}$ \\
\hline
\end{tabular}


obtained frameworks when compared to that of nickelchromium cast copings (2).

In order to minimize external variations associated to any experimental work, each wax pattern was modeled over plastic copings by a single operator. Moreover, since possible wax volumetric changes may occur in this step, the wax-ups were randomly assigned to the experimental groups of alloys in order to eliminate bias. Afterwards, the investing and casting procedures were carried out by the same specialized technician under the authors' supervision, following the manufacturers' recommendations.

Multiple factors related to the investing and casting procedures may result in imperfect structures showing roughness, distortion, incomplete/missing casts, marginal discrepancies, and/or porosity defects; including the investment convenience for the alloy selected, the liquid-powder ratio of the investment material, the investing technique, air bubble entrapments, rapid heating rates, underheating or overheating, melting temperature, casting pressure, impact of molten alloy, and carbon inclusions (19). Furthermore, in spite of great care being taken to standardize procedures, any process involving manipulation of diverse materials is technique sensitive and individual aberrations can occur despite the technician experience.

A customized tool was used for fixing all the bridge structures under a constant seating pressure, taking into account that the lack of passive fit may intensify the mechanical fatigue and plastic distortion in cemented structures under occlusal loads (3). Additionally, the implant-replicas were embedded inside composite cubes to reproduce the mean elastic module reported for trabecular human bone, which allows for some of the forces to be dissipated (20).

Up to date, there have not been established minimum castability requirements for the different marketed dental alloys that would ensure satisfactory metal-ceramic restorations $(6,19)$. Therefore, further investigation is needed to determine the importance of a precise management of investment and casting technologies that could improve the quality of the obtained substructures.

Within the limitations of this study, it may be concluded that fracture strength of metallic frameworks depended on the alloy type and investing and casting procedures. Cobalt-chromium casts demonstrated significant porosities inside the connectors, which strongly decreased the fracture resistance. Therefore, an accurate casting of titanium with a pressure-differential system may result in the most predictable technique under the tested experimental conditions, even when failure loads of all tested groups exceeded the habitual biting forces.

\section{References}

1. Cheng WW, Ju CP, Lin JH. Structure, castability and mechanical properties of commercially pure and alloyed titanium cast in graphite mould. J Oral Rehabil. 2007;34:528-40.

2. Oyagüe RC, Turrión AS, Toledano M, Monticelli F, Osorio R. In vitro vertical misfit evaluation of cast frameworks for cement-retained implant-supported partial prostheses. J Dent. 2009;37:52-8.

3. Karl M, Taylor TD, Wichmann MG, Heckmann SM. In vivo stress behavior in cemented and screw-retained five-unit implant FPDs. J Prosthodont. 2006;15:20-4.

4. Hjalmarsson L. On cobalt-chrome frameworks in implant dentistry. Swed Dent J Suppl. 2009;201:3-83.

5. Morris HF. Properties of cobalt-chromium metal ceramic alloys after heat treatment. J Prosthet Dent. 1990;63:426-33.

6. Leal MB, Paulino SM, Pagnano VO, Bezzon OL. Influence of investment type and sprue number on the casting accuracy of titanium crown margins. J Prosthet Dent. 2006;95:42-9.

7. Jang KS, Youn SJ, Kim YS. Comparison of castability and surface roughness of commercially pure titanium and cobalt-chromium denture frameworks. J Prosthet Dent. 2001;86:93-8.

8. De Oliveira Correa G, Henriques GE, Mesquita MF, Sobrinho LC. Over-refractory casting technique as an alternative to onepiece multi-unit fixed partial denture frameworks. J Prosthet Dent. 2006;95:243-8.

9. Hart CN, Wilson PR. Evaluation of welded titanium joints used with cantilevered implant-supported prostheses. J Prosthet Dent. 2006;96:25-32.

10. Ulusoy M, Toksavul S. Fracture resistance of five different metal framework designs for metal-ceramic restorations. Int J Prosthodont. 2002;15:571-4.

11. Yildirim M, Fischer H, Marx R, Edelhoff D. In vivo fracture resistance of implant-supported all-ceramic restorations. J Prosthet Dent. 2003;90:325-31.

12. Sabbagh J, Vreven J, Leloup G. Dynamic and static moduli of elasticity of resin-based materials. Dent Mater. 2002;18:64-71.

13. Rho JY, Tsui TY, Pharr GM. Elastic properties of human cortical and trabecular lamellar bone measured by nanoindentation. Biomaterials. 1997:18:1325-30.

14. Kokubo Y, Tsumita M, Sakurai S, Torizuka K, Vult von Steyern P, Fukushima S. The effect of core framework designs on the fracture loads of all-ceramic fixed partial dentures on posterior implants. J Oral Rehabil. 2007;34:503-7.

15. Wictorin L, Julin P, Möllersten L. Roentgenological detection of casting defects in cobalt-chromium alloy frameworks. J Oral Rehabil. 1979;6:137-46.

16. Bessing C, Bergman M. The castability of unalloyed titanium in three different casting machines. Swed Dent J. 1992;16:109-13.

17. Anusavice KJ, Kakar K, Ferree N. Which mechanical and physical testing methods are relevant for predicting the clinical performance of ceramic-based dental prostheses? Clin Oral Implants Res. 2007;18 Suppl 3:218-31.

18. Zarone F, Sorrentino R, Traini T, Di lorio D, Caputi S. Fracture resistance of implant-supported screw- versus cement-retained porcelain fused to metal single crowns: SEM fractographic analysis. Dent Mater. 2007;23:296-301.

19. Paulino SM, Leal MB, Pagnano VO, Bezzon OL. The castability of pure titanium compared with Ni-Cr and Ni-Cr-Be alloys. J Prosthet Dent. 2007;98:445-54.

20. Jacques LB, Moura MS, Suedam V, Souza EA, Rubo JH. Effect of cantilever length and framework alloy on the stress distribution of mandibular-cantilevered implant-supported prostheses. Clin Oral Implants Res. 2009;20:737-41.

\section{Acknowledgments}

This investigation was supported by CICYT/FEDER MAT200802347/MAT, JA-P07-CTS-2568, JA-P08-CTS-3944, and UNGR08-1E-030. 